\title{
Erratum to: A Phase 1 dose-escalation study of the safety and pharmacokinetics of once-daily oral foretinib, a multi-kinase inhibitor, in patients with solid tumors
}

\author{
Geoffrey I. Shapiro $^{1} \cdot$ Stewart McCallum ${ }^{2}$ - Laurel M. Adams ${ }^{3,4}$ - Laurie Sherman ${ }^{5,16}$.

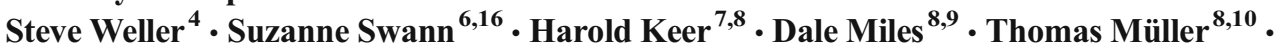 \\ Daniel C. Rabe ${ }^{11,17} \cdot$ Fabiola Cechi $^{12,17}$ - Donald P. Bottaro ${ }^{13}$ - Patricia LoRusso ${ }^{14,15}$
}

Published online: 26 September 2015

(C) Springer Science+Business Media New York 2015

\section{Erratum to: Invest New Drugs (2013) 31:742-750 DOI 10.1007/s10637-012-9881-z}

In the original version of this article, three authors are missing. The complete list of authors and their current and former (at the time the work was performed) affiliations are provided. The additional authors declare no conflicts of interest.

In addition, pharmacodynamics assays performed to obtain absolute plasma concentrations of HGF, VEGF, soluble $\mathrm{KDR}$ and soluble Met as described in the original article were incorrectly attributed in the Methods section.

The following text...

"Absolute plasma concentrations of HGF, VEGF, soluble KDR and soluble Met were determined by two-site electrochemiluminescent immunoassay as described previously [1]."

The online version of the original article can be found at $\mathrm{http}: / / \mathrm{dx}$.doi.org/ 10.1007/s10637-012-9881-z.

Laurel M. Adams

adamsla@medimmune.com

1 Present address: Dana-Farber Cancer Institute and Harvard Medical School, Boston, MA, USA

2 Present address: GlaxoSmithKline, Collegeville, PA, USA

3 Present address: Medimmune, Gaithersburg, MD, USA

4 Oncology Research and Development, GlaxoSmithKline, Research Triangle Park, Durham, NC, USA

5 Present address: Janssen Research \& Development, Spring House, PA, USA

6 CSL Behring, King of Prussia, PA, USA

7 Present address: Astex Pharmaceuticals, Inc., Pleasanton, CA, USA

8 Exelixis, South San Francisco, CA, USA ...replaces the original text below:

"Plasma levels of soluble MET (sMET), HGF, soluble VEGFR2 (sVEGFR2), and VEGF-A were measured using enzyme-linked immunosorbent assay at Pathway Diagnostics, Malibu, California, USA (now Quest Diagnostics Biomarker Lab, Valencia, California, USA) and at Exelixis (for sMET)." The following citation refers to the text added, above.

[1] Athauda G, Giubellino A, Coleman JA, Horak C, Steeg PS, Lee MJ, Trepel J, Wimberly J, Sun J, Coxon A, Burgess TL, Bottaro DP. c-Met ectodomain shedding rate correlates with malignant potential. Clin Cancer Res 2006;12:4154-62.

Finally, the following acknowledgement statement should be added:

"This work was supported in part by the Intramural Research Program of the NIH, National Cancer Institute, Center for Cancer Research."

9 Present address: Genentech, San Francisco, CA, USA

10 Present address: Biomarker, Translational \& Predictive Medicine Consulting, San Francisco, CA, USA

11 Present address: University of Chicago, Chicago, IL, USA

12 Present address: NantOmics, LLC, Rockville, MD, USA

13 Present address: Urologic Oncology Branch, Center for Cancer Research, National Cancer Institute, Bethesda, MD, USA

14 Present address: Yale Cancer Center, New Haven, CT, USA

15 Barbara Ann Karmanos Cancer Institute/Wayne State University, Detroit, MI, USA

16 GlaxoSmithKline, Collegeville, PA, USA

17 Urologic Oncology Branch, Center for Cancer Research, National Cancer Institute, Collegeville, PA, USA 